\title{
Devenons-nous les disciples de notre confrère Knock?
}

Au sujet de l'article de G. Domenighetti paru en janvier, nous avons reçu tardivement les considérations ci-dessous: elles ont suivi quelques méandres électroniques, mais nous nous faisons un plaisir de les publier, même avec retard. risques diffus», des «conflits d'intérêts qui lient l'industrie pharmaceutique aux chercheurs et aux universités, ainsi qu'aux membres des conférences de consensus» (cf. à cet égard les prises de positions remarquables de l'éminente hospitalouniversitaire américaine Marcia Angell [2] et les efforts de l'Académie suisse des sciences médicales et de la FMH [3]); se préoccuper aussi de la «surmédicalisation de la vie par l'augmentation des conditions qui pourront être définies comme des maladies» (p. 222 et 223 de l'article). Ceci pour arriver à la «construction industrielle des maladies». Dans ces évolutions, c'est un fait de la vie qu'il y a une implication des médecins, qu'elle soit passive ou active.

Quelques exemples ces dernières années: la promotion omniprésente du Viagra (devenu pratiquement un nom commun) et des médicaments concurrents, par des encarts publicitaires qui, sous couvert d'aider le mâle sous-performant en lui proposant d'appeler une source de bon conseil, s'attache avec beaucoup de détermination - et de moyens! - à convaincre les Suisses que: «Si vous êtes un homme, vous avez (forcément) des problèmes d'érection». Il y a trois ans je pense, j'ai été interpellé en gare de Lausanne par une charmante personne distribuant à tout un chacun un prospectus qui avait le propos, selon ses concepteurs (soutenus par un professeur de médecine nommément désigné), d'apporter une information/éducation sur les brûlures d'estomac. Message sous-jacent d'une telle opération - c'est en tout cas ainsi que je l'ai vécu: «Si vous êtes un être humain, vous devez avoir des troubles gastriques et il importe que vous soyez médiqués pour cela.»

Les résultats pour la Suisse d'une étude européenne récente sur la douleur, financée par l'industrie pharmaceutique, montrent que, dans un ménage sur trois, il y a une personne souffrant de douleurs. Un Suisse sur six présente des douleurs chroniques et il en souffre, en moyenne, depuis huit ans. Des réponses des personnes interviewées, on tire la conclusion que ce problème est largement sous-estimé et sous-traité. C'est possible. Cela étant, la dissémination publique de ces résultats ne correspond-elle pas aussi à dire à la population, avec une certaine insistance, «il est probable que vous n'absorbez pas assez d'antalgiques, et pas assez souvent». 
La réflexion est requise aussi à propos d'autres exemples de médicalisation moderne de la vie, par exemple le remplacement, maintenant déjà et de manière accrue à l'avenir, des mécanismes naturels de procréation par des variantes technicisées, par exemple la césarienne à la demande [4].

Tout au long d'une carrière de santé publique, je me suis engagé pour une information adéquate, suffisante et compréhensible, du public en ce qui concerne sa santé et la prévention de risques pour celle-ci. J'ai trouvé que c'était une belle mission. Mais quand la société de l'information dans laquelle nous sommes entrés transmet des messages orientés et présentés de façon à promouvoir la consommation en excès de prestations médico-sanitaires, et spécialement celle de médicaments, il y a lieu d'examiner très attentivement la situation et de contrer ces dérives.

En soulignant ici une chose: lesdits messages peuvent être basés sur des données objectives (prévalence d'un trouble, observation d'un paramètre biologique ou d'un résultat d'imagerie en dehors de la norme); mais ils négligent opportunément de dire que, souvent, on peut vivre très longtemps sans dommage et sans souci avec l'altération en question et mourir d'autre chose (Domenighetti cite plusieurs exemples connus).
Ces messages sont aussi fort discrets quant aux effets secondaires potentiellement sérieux, y compris le fait d'alarmer la personne (problème des faux positifs), de la pousser indûment vers un «état de malade».

Notre confrère Knock disait qu'une personne en bonne santé est un malade qui s'ignore. Bien qu'il soit manifeste que nous n'y échappons pas, il serait regrettable que nous suivions trop fidèlement son exemple... De plus, on était à cet égard, du temps de Jules Romains, dans l'artisanat. On a depuis lors beaucoup progressé dans la fabrication industrielle de malades.

Dr Jean Martin, Echandens

\section{Références}

1 Domenighetti G. Grandeur et misère des systèmes universels de santé. Bull Méd Suisses 2005;86(4): 221-6.

2 Angell M. The Truth about the Drug Companies. New York: Random House; 2004.

3 ASSM et FMH. Collaboration corps médical industrie. Directives mises en consultation. Bull Méd Suisses 2005;86(2):115-21.

4 Martin J. Biomédecine et procréation - Quel avenir pour les variantes technicisées de la manière de faire des enfants dont nous avons l'habitude? Revue médicale suisse 2005;1:453-8. 